\title{
REGULAR NONNEGATIVE MATRICES ${ }^{1}$
}

\author{
ROBERT J. PLEMMONS
}

\begin{abstract}
Regular elements in the semigroup $\mathscr{N}_{n}$ of all $n \times n$ nonnegative matrices are characterized. The regular $\mathscr{D}$-classes in $\mathscr{N}_{n}$ are investigated and an alternate proof is given for a theorem of Flor characterizing the maximal subgroups.
\end{abstract}

I. Introduction. Several authors in recent years have investigated the algebraic structure of semigroups of nonnegative matrices. In particular, maximal subgroups of such semigroups have been thoroughly studied. Brown [2] has shown that such subgroups are finite if compact and Flor [4] has a simple proof of the Brown result. Let $\mathscr{N}_{n}$ denote the semigroup of all $n \times n$ nonnegative matrices, let $\mathscr{S}_{n}$ denote the $n \times n$ stochastic matrices and let $\Omega_{n}$ denote the $n \times n$ doubly stochastic matrices. Flor has also shown that the maximal subgroups of $\mathscr{N}_{n}$ are isomorphic to complete nomomial groups over the reals. Schwarz [7] has characterized the maximal subgroups of $\mathscr{S}_{n}$ as groups isomorphic to full symmetric groups while he [8] and Farahat [3] have independently shown that the maximal subgroups of $\Omega_{n}$ are isomorphic to direct products of full symmetric groups.

In [5] the Green's relations and regularity in the semigroup $\Omega_{n}$ of doubly stochastic matrices were investigated. The purpose of this note is to characterize regularity in $\mathscr{N}_{n}$ and to provide an alternate proof of the Flor result concerning the maximal subgroups. The techniques used here involve only elementary matrix properties and elementary semigroup theory.

II. Regularity. The following concepts from the algebraic theory of semigroups will be used. The definitions and notation follow those in [1]. Let $T$ denote a semigroup and let $a, b \in T$. Then the relation $\mathscr{R}[\mathscr{L}]$ is defined on $T$ by the rule $a \mathscr{R} b[a \mathscr{L} b]$ if and only if $a$ and $b$ generate the same principal right [left] ideal in $T$. The relation $\mathscr{H}$ is defined to be $\mathscr{L} \cap \mathscr{R}$.

Received by the editors September 18, 1972.

AMS (MOS) subject classifications (1970). Primary 20M99; Secondary 15A48, $20 \mathrm{G} 20$.

Key words and phrases. Groups, nonnegative matrices, regularity in semigroups of nonnegative matrices.

${ }^{1}$ Research supported in part by NSF Grant GP-15943.

(C) American Mathematical Society 1973 
Then each of $\mathscr{R}, \mathscr{L}$ and $\mathscr{H}$ are equivalence relations on $T$. The intersection of all the equivalence relations on $T$ containing the union $\mathscr{R} \cup \mathscr{L}$ is denoted by $\mathscr{D}$. These are known as the Green's relations on $T$ and they play a fundamental role in the study of the algebraic structure of semigroups (see [1, Chapter II] for a complete discussion).

An element $a$ in the semigroup $T$ is said to be regular in $T$ if the equation $a=a x a$ is solvable for $x \in T$. If in addition $x=x a x$ then $a$ and $x$ are said to be semi-inverses of each other. Notice that if $a=a x a$, then $a$ and $x a x$ are semi-inverses. If an element in a $\mathscr{D}$-class $D$ of $T$ is regular then each element in $D$ is regular and $D$ is called a regular $\mathscr{D}$-class in $T$. In this case there is associated with $D$ a maximal subgroup of $T$, isomorphic to each $\mathscr{H}$-class of $T$ in $D$ that contains an idempotent. Moreover, every maximal subgroup of $T$ is obtained in this way [1].

Clearly not every matrix in the semigroup $\mathscr{N}_{n}$ of nonnegative matrices is regular. An $n \times n$ matrix $A$ of rank $r$ will be called $r$-monomial if $A$ has rank $r$ and each row and each column of $A$ contains at most one nonzero entry. If $r=n$ then $A$ will be called monomial. The only regular nonsingular matrices in $\mathscr{N}_{n}$ are the nonnegative monomial matrices.

Now let $I_{r}$ denote the identity matrix of order $r$. If $A$ is an $r \times n[n \times r]$ matrix of rank $r$, then any solution $X$ to $A X=I_{r}\left[X A=I_{r}\right]$ is called a right [left] inverse of $A$. For an $n \times n$ matrix $A$ of rank $r$, there exist $n \times r$ and $r \times n$ matrices $B$ and $G$, respectively, such that

$$
A=B G \text {. }
$$

In this case (1) is called a rank factorization of $A$. If $B$ and $G$ are nonnegative, then (1) will be called a nonnegative rank factorization. It follows that if $B_{L}$ is any left inverse of $B$ and $G_{R}$ is any right inverse of $G$, then

$$
X=G_{R} B_{L}
$$

is a semi-inverse of $A$. Conversely, every semi-inverse of $A$ is obtained in this way. This leads to the following lemma.

Lemma 1. Let $A \in \mathscr{N}_{n}$ and suppose that $A=B G$ is a nonnegative rank factorization. Then $A$ is regular in $\mathscr{N}_{n}$ if and only if $B$ and $G$ have nonnegative left and right inverses, respectively.

Proof. If $A$ is regular and $X$ is a semi-inverse of $A$ in $\mathscr{N}_{n}$, then $X$ has the form (2) for some $B_{L}$ and $G_{R}$. In this case $B_{L}=I_{r} B_{L}=G G_{R} B_{L}=G X$ which is nonnegative. Dually $G_{R}$ is nonnegative. The converse is immediate.

In order to investigate regularity in $\mathscr{N}_{n}$, it is thus important to know when a nonnegative matrix has a nonnegative right [left] inverse. 
LEMMA 2. Let $A$ be an $r \times n$ nonnegative matrix of rank $r$. Then $A$ has $a$ nonnegative right inverse if and only if $A$ has a monomial submatrix of order $r$. In this case $A$ has a nonnegative right inverse with $r$ nonzero entries.

Proof. Suppose that $X$ is an $n \times r$ nonnegative matrix such that $A X=$ $I_{r}$. That is

$$
\sum_{k=1}^{n} a_{i k} x_{k j}=0 \quad \text { if } i \neq j,
$$

for each $i, j$ where $1 \leqq i, j \leqq r$. Then for each $i$ there exists some $k$ such that $a_{i k} \neq 0$ and $a_{l k}=0$ for $l \neq i, 1 \leqq l \leqq r$. That is, the $k$ th column of $A$ has exactly one nonzero entry and that entry is in the $i$ th row. Since $A$ has rank $r$ and since $r$ columns of $A$ have precisely one nonzero entry, $A$ has a monomial submatrix of order $r$.

For the converse let $P$ be a permutation matrix of order $n$ such that $A P=(B, C)$ where $B$ is monomial of order $r$ and let

$$
Y=\left(\begin{array}{c}
B^{-1} \\
0
\end{array}\right) \text {. }
$$

Then $X=P Y$ has the desired properties.

Notice that a result dual to Lemma 2 can be stated for left inverses.

Now any nonnegative rank 1 matrix $A$ has a nonnegative rank factorization. In particular there exist nonnegative column $n$-vectors $x$ and $y$ such that $A=x y^{T}$. Suppose that $A$ has rank $r$ and has the partitioned row block form

$$
A=\left(\begin{array}{c}
H_{1} \\
\cdot \\
\cdot \\
H_{r} \\
0
\end{array}\right)
$$

where each $H_{i}$ has rank 1 and where the zero block may not appear. Then for $A$ nonnegative there exist nonnegative vectors $x^{i}$ and $y^{i}$ such that $H_{i}=x^{i}\left(y^{i}\right)^{T}$ and $A$ has the nonnegative rank factorization

$$
A=\left(\begin{array}{cccc}
x^{1} & 0 & \cdots & 0 \\
0 & x^{2} \cdot & & \cdot \\
\cdot & & \cdot & \cdot \\
\cdot & & \cdot & \cdot \\
0 & & & \cdot \\
x^{r}
\end{array}\right)\left(y^{1} y^{2} \cdots y^{r}\right)^{T} .
$$


This fact will be used in the proof of the main result in this section. Another useful tool is the following result due to Flor.

LEMMA 3 (FLOR [4, THEOREM 2]). A nonnegative matrix $E$ of rank $r$ is idempotent if and only if there exists a permutation matrix $P$ such that $P E P^{T}$ has the form

$$
P E P^{T}=\left(\begin{array}{cccc}
J & J U & 0 & 0 \\
0 & 0 & 0 & 0 \\
V J & V J U & 0 & 0 \\
0 & 0 & 0 & 0
\end{array}\right)
$$

where $J$ has the form

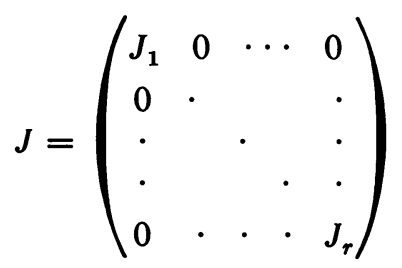

and the $J_{i}$ are nonnegative idempotent matrices of rank 1 and where $U$ and $V$ are nonnegative matrices of the appropriate sizes.

THEOREM 1. Let $A$ be an $n \times n$ nonnegative matrix of rank $r$. Then the following statements are equivalent.

(i) $A$ is regular in $\mathscr{N}_{n}$.

(ii) $A$ has a semi-inverse in $\mathscr{N}_{n}$ of the form $D_{1} A^{T} D_{2}$ where $D_{1}$ and $D_{2}$ are nonnegative diagonal matrices.

(iii) $A$ has a semi-inverse in $\mathscr{N}_{n}$ of r-monomial type.

(iv) A has a monomial submatrix of order $r$.

Proof. Assume that $A$ is regular in $\mathscr{N}_{n}$ and let $X$ be a nonnegative inverse of $A$. For $E=A X$, choose a permutation matrix $P$ such that $K=$ $P E P^{T}$ has the form (5). Then $Y=X P^{T}$ is a semi-inverse of $C=P A$ in $\mathscr{N}_{n}$. Next partition $C$ into the row block form

$$
C=\left(\begin{array}{l}
H \\
L \\
M \\
N
\end{array}\right)
$$

corresponding to the row block form of $K$. Now $K C=P E P^{T} P A=P E A=$ $P A=C$ and thus $J H=H, L=0, V J H=V H=M$ and $N=0$. Thus $C$ has 
the form

$$
C=\left(\begin{array}{c}
H \\
0 \\
V H \\
0
\end{array}\right)
$$

Partition the matrix $H$ into the row block form

$$
H=\left(\begin{array}{c}
H_{1} \\
\cdot \\
\cdot \\
\cdot \\
H_{r}
\end{array}\right)
$$

corresponding to the partitioned form of $J$ given in (6). From $J H=H$ it follows that $J_{i} H_{i}=H_{i}$ for each $i$. Thus $H_{i}$ has rank 1 . Then by the remarks preceding Lemma $3, H$ has a nonnegative rank factorization $H=B_{1} G$. Let

$$
B_{2}=\left(\begin{array}{c}
B_{1} \\
0 \\
V B_{1} \\
0
\end{array}\right)
$$

Then $C=B_{2} G$ is a nonnegative rank factorization of $C$. Moreover for $B=P^{T} B_{2}, A=B G$ is a nonnegative rank factorization of $A$. Then by Lemma $1, B$ and $G$ have nonnegative left and right inverses, respectively. By Lemma 2 and its dual, $B_{L}$ and $G_{R}$ can be chosen to have exactly $r$ nonzero entries. For this choice, the matices $D_{1}=G_{R} G_{R}^{T}$ and $D_{2}=B_{L}^{T} B_{L}$ are $n \times n$ nonnegative diagonal matrices and

$$
\begin{aligned}
D_{1} A^{T} D_{2} & =G_{R} G_{R}^{T}(B G)^{T} B_{L}^{T} B_{L}=G_{R}\left(G G_{R}\right)^{T}\left(B_{L} B\right)^{T} B_{L} \\
& =G_{R} I_{r}^{2} B_{L}=G_{R} B_{L},
\end{aligned}
$$

so that $D_{1} A^{T} D_{2}$ is a nonnegative semi-inverse of $A$. This establishes (ii). Since in this case $G_{R} B_{L}$ is of $r$-monomial type, (i) also implies (iii).

Next assume (iii) holds and let $X$ be a semi-inverse of $A$ in $\mathscr{N}_{n}$ of $r$ monomial type. Then there exist permutation matrices $P$ and $Q$ so that $Y=P X Q$ has block form

$$
Y=P X Q=\left(\begin{array}{ll}
M & 0 \\
0 & 0
\end{array}\right)
$$


where $M$ is an $r \times r$ monomial matrix. Then $Y$ is a semi-inverse of $B=$ $Q^{T} A P^{T}$ in $\mathscr{N}_{n}$ so that $B$ has the block form

$$
B=\left(\begin{array}{ll}
B_{1} & B_{2} \\
B_{3} & B_{4}
\end{array}\right)
$$

where $B_{1}=M^{-1}$ and $B_{4}=B_{3} M B_{2}$. Thus $A=Q B P$ has a monomial submatrix of order $r$, establishing (iv). The proof that (iv) implies (iii) is obtained by retracing these steps. Since statements (ii) and (iii) each imply (i) trivially, the proof of the theorem is complete.

III. Maximal subgroups. The $\mathscr{D}$-class containing the zero matrix 0 in $\mathscr{N}_{n}$ is $\{0\}$ while the $\mathscr{D}$-class containing $I_{n}$ consists of the group of all monomial matrices in $\mathscr{N}_{n}$. The following result gives a complete description of all the regular $\mathscr{D}$-classes. It will be used to establish the maximal subgroup characterization.

TheOREM 2. Let $A \in \mathscr{V}_{n}$ be regular of rank $r$ and let $D$ denote the $\mathscr{D}$ class containing $A$. Then $D$ contains the canonical idempotent

$$
E=\left(\begin{array}{ll}
I_{r} & 0 \\
0 & 0
\end{array}\right) .
$$

Moreover, $D$ consists of all members of $\mathscr{N}_{n}$ of rank $r$ containing a monomial submatrix of order $r$.

Proof. Since $A$ is regular, it has an $r$-monomial semi-inverse $X$ in $\mathscr{N}_{n}$ by Theorem 1. From [1, Theorem 2.18], it follows that $X \in D$. Let $P, Q$ be permutation matrices such that $Y=P X Q$ has the form (8), where $M$ is monomial of order $r$. Then $Y \in D$ so that

$$
Z=\left(\begin{array}{cc}
M^{-1} & 0 \\
0 & 0
\end{array}\right)
$$

is a semi-inverse of $Y$ in $D$. Thus the canonical idempotent $E=X Z$ belongs to $D$. By this argument, $D$ contains all regular members of $\mathscr{N}_{n}$ having rank $r$. By Theorem 1 these matrices have a monomial submatrix of order $r$. Clearly each member of $D$ has this property.

Notice that by Theorem $2, \mathscr{N}_{n}$ has exactly $n+1$ regular $\mathscr{D}$-classes. Moreover the maximal subgroup of $\mathscr{N}_{n}$ associated with the $\mathscr{D}$-class $D$ is isomorphic to the $\mathscr{H}$-class, $H$, containing $E$. But $H$ is isomorphic to the complete monomial group of degree $r$ over the reals [6]. This provides an alternate proof of the following result first given by Flor [4]. 
COROLlaRy 1. The maximal subgroups of $\mathscr{N}_{n}$ are isomorphic to the complete monomial groups of degree $r$ over the reals, $0 \leqq r \leqq n$.

\section{REFERENCES}

1. A. H. Clifford and G. B. Preston, The algebraic theory of semigroups. Vol. 1, Math. Surveys, no. 7, Amer. Math. Soc., Providence, R.I., 1961. MR 24 \#A2627.

2. D. R. Brown, On clans of non-negative matrices, Proc. Amer. Math. Soc. 15 (1964), 671-674. MR 29 \#2786.

3. H. K. Farahat, The semigroup of doubly-stochastic matrices, Proc. Glasgow Math. Assoc. 7 (1966), 178-183. MR 34 \#2590.

4. P. Flor, On groups of non-negative matrices, Compositio Math. 21 (1969), 376-382. MR 41 \#1769.

5. J. S. Montague and R. J. Plemmons, Doubly stochastic matrix equations, Israel J. Math. (to appear).

6. O. Ore, Theory of monomial groups, Trans. Amer. Math. Soc. 51 (1942), 15-64. MR 3, 197.

7. S. Schwarz, On the structure of the semigroup of stochastic matrices, Magyar Tud. Akad. Mat. Kutató Inst. Közl. 9 (1964), 297-311. MR 32 \#5776.

8. - A note on the structure of the semigroup of doubly-stochastic matrices, Mat. Casopis Sloven. Akad. Vied. 17 (1967), 308-316. MR 39 \#2791.

Centre de Recherches Mathematiques, Université de Montréal, Montréal, QUÉBEC, CANADA

Departments of Mathematics and Computer Science, University of Tennessee, Knoxville, Tennessee 37916 